\title{
Die Anwendung der Traditionellen Chinesischen Medizin (TCM)/Akupunktur in der Therapie und Prävention von SARS-CoV-2-Infektionen
}

\author{
White Paper des Wissenschaftszentrums der deutschen Ärztegesellschaft für Akupunktur
}

\begin{abstract}
Die Infektion mit SARS-CoV-2 und der damit verbundenen COVID-19-Erkrankung belastet gegenwärtig die medizinischen, sozialen und ökonomischen Strukturen weltweit. In diesem Kontext erschienen jüngst mehrere laienhafte, aber auch wissenschaftliche Abhandlungen zur Rolle der Traditionellen Chinesischen Medizin (TCM)/Akupunktur.
\end{abstract}

\section{》) Der TCM/Akupunktur kommt eine allgemein-unterstützende Rolle in der antiviralen Therapie zu}

Vorab sei an dieser Stelle klargestellt, dass der TCM/Akupunktur keine primäre, sondern eine unterstützende Rolle in der antiviralen Therapie und ein Einsatz zur Symptomlinderung zukommt.

Es kursieren Arbeiten die den Stellenwert der Akupunktur, aber auch der chinesischen Arzneimitteltherapie in der Therapie schwerer inflammatorischer Zustände untersuchen. Eine aktuelle Metaanalyse zeigt, dass die Akupunktur am Punkt Magen 36 zu einer Reduktion von Organschädigungen nach Sepsis unterschiedlicher Ursache in experimentellen Tiermodellen beiträgt [3]. Als Mechanismen diskutieren die Autoren, dass durch Akupunktur oxidativer Stress und Entzündungsprozesse reduziert, sowie die Mikrozirkulation der Gewebe verbessert werden.

Eine weitere Metaanalyse beschäftigt sich mit der Wirksamkeit des patentierten Injektionsmittels Xuebijing bei 1144 Patienten mit Sepsis [5]. Die Autoren berichten, dass die Substanz die 28-tägige Mortalitätsrate, die APACHE II Scores ${ }^{1}$, die Zahl der weißen Blutzellen und die Körpertemperaturen der Patienten verbessert [5]. Als einschränkend für die Sicherheit der Aussage gelten ein hohes Verzerrungsrisiko und eine niedrige Qualität der eingeschlossenen Studien. Präklinische Daten zu der

${ }^{1}$ Acute Physiology and Chronic Health Evaluation APACHE, auf Intensivstationen verwendetes Verfahren zur Vorhersage der Überlebenswahrscheinlichkeit von Patienten.

Deutsche Zeitschrift für Akupunktur 2020 • 63 (2): 70-73

https://doi.org/10.1007/s42212-020-00271-7

Online publiziert: 8 . April 2020

๑) Springer Medizin Verlag GmbH, ein Teil von Springer Nature 2020
Substanz sind nicht transparent verfügbar. Jedoch wurde in der Vergangenheit bereits eine Vielzahl chinesischer Arzneimittel postuliert (teilweise von der chinesischen Arzneimittelbehörde zugelassen), die nachgewiesenermaßen das Eindringen von Viren in die Wirtszelle, deren Replikation, Zusammensetzung sowie letztlich Freisetzung aus der Zelle beeinflussen können [6].

Bereits während der SARS-Epidemie 2002/2003 skizzierte eine Übersicht chinesischer Publikationen den Nutzen der TCM [4]. Insbesondere der chinesischen Arzneitherapie wurden positive Effekte in den Aspekten besserer Temperaturkontrolle, seltenerer Infektionen des Thorax, niedrigeren Steroidbedarfs und genereller Symptomlinderung zugeschrieben.

Eine weitere Metaanalyse über 8 Studien $(n=488)$ beschreibt die Reduktion der Mortalität und Fieberdauer und Abnahme steroidverursachter Nebenwirkungen und pathologischer Röntgen-Thorax-Befunde, jeweils in Kombination mit konventionellen Maßnahmen bei SARS-Patienten [8].

Eine Cochrane-Analyse aus dem Jahr 2012 konnte keine Reduktion der Mortalität von SARS durch die Kombination von chinesischer Arzneimitteltherapie und westlicher Medizin zeigen [9]. Es existieren Hinweise für eine verbesserte Symptomatik und Lebensqualität, die Abnahme pulmonaler Infiltrate sowie eine niedrigere benötigte Steroiddosis. Die Qualität der Studien ist niedrig, und Langzeitbeobachtungen fehlen [9].

Betreffend die Infektionen mit SARS-CoV-2 finden sich derzeit zahlreiche Berichte in Wissenschaftsjournalen zu den Perspektiven der TCM $[7,10,12]$. Besonders ausführlich ist eine international gelistete Übersichtsarbeit der Universität in Macau [16]. Die Autoren geben an, dass der Anteil an infizierten Patienten in China, die sich zusätzlich mit TCM behandeln lassen, bei über $85 \%$ liegt. In manchen Provinzen wurde berichtet, dass über $90 \%$ der Patienten zusätzlich mit TCM behandelt wurden [13]. Das Spektrum der konventionellen Therapie umfasst derzeit die Sauerstoffzufuhr, Antibiotikagabe, antivirale Medikation, Steroide sowie die Plasmatherapie. Da seitens der WHO bisher keine ,spezifische Therapie zur Prävention und Behandlung von SARS-CoV-2" verfügbar ist, sehen die Autoren der Universität Macau es als gerechtfertigt, insbesondere den Einsatz chinesischer Arzneitherapien, die seit vielen Dekaden zur Kontrolle von Infektionskrankheiten angewandt wurden, als wertvolle Ergänzung zu betrachten [16]. In der Begründung potenziell wirksamer Substanzen argumentieren die Autoren insbesondere mit den bekannten antiviralen Aktivitäten von Arzneimitteln, welche 
aus der SARS-Epidemie bekannt sind und zahlreiche gemeinsame molekulare Andockstrukturen bei SARS-CoV-2 aufweisen. Einschränkend ist hier, dass die Kenntnis über die direkte molekulare Wirkung in SARS-CoV-2 aussteht.

\section{I) Chinesische Gesundheitsbehörden empfehlen die Kombination der TCM mit den konventionellen Maßnahmen}

Die Gesundheitsbehörden von 26 chinesischen Provinzen empfehlen (Stand 17.02.2020) die Kombination der TCM mit den konventionellen Maßnahmen [16]. Gemäß den Autoren finden sich je nach Provinz unterschiedlich angewendete Rohdrogen. Die Nationale Gesundheitskommission der Volksrepublik China stellt in ihrer Leitlinie zur Diagnose und Therapie von 2019-nCoV-infizierten Pneumonien (7. Version; [1, 15]) folgende Präparate in den Vordergrund (Tab. 1).

Bisher stützen sich diese Empfehlungen nicht auf klinische Studien. Jedoch war die Coronapandemie Anlass dazu, dass zum 01.03.2020 bei der Nationalen Gesundheitskommission der Volksrepublik China 50 klinische Studien bei COVID-19-erkrankten Patienten registriert waren, welche die Wirksamkeit und Sicherheit der TCM (insbesondere chinesischer Arzneimittel) untersuchen [16]. Im amerikanischen Studienregister clinicaltrials.gov finden sich gegenwärtig 9 Einträge zu Studien mit chinesischen Arzneimitteln. Zu keiner dieser Studien wurden bisher Ergebnisse veröffentlicht.

Bereits vorhandene Studienergebnisse mit SARS-CoV-2-positiven Patienten wurden vor allem in chinesischsprachigen Publikationsorganen veröffentlicht. So berichten Yang et al. [16] von retrospektiven Beobachtungen, die zeigen, dass durch die unterschiedlichen Konzepte in den Provinzen in 30-60 \% der Fälle die Schwere von Symptomen wie Fieber und Husten gelindert werden konnte.
In westlichen Wissenschaftsdatenbanken finden sich aktuell diverse Fallberichte. Deng et al. [2] berichten über einen Patienten mit klinischem Verdacht einer schweren COVID-19-Pneumonie, welcher drei Tage lang mit einer Immunglobulintherapie behandelt wurde. Anschließend wurde er zweimal täglich mit Moxibustionskästchen an den Punkten LG 14, KG 4 und beidseits Ma 36 behandelt. Darunter stieg die Zahl der weißen Blutkörperchen. Durch die Gabe chinesischer Arzneimitteltherapie (2 Dekokte: Ma Xing Shi Gan und Xiao Chai $\mathrm{Hu}$ ) konnte die Körpertemperatur besser kontrolliert werden. Ab dem neunten Tag war die Dyspnoe deutlich reduziert und der Patient benötigte keine weitere Sauerstoffzufuhr. Nach dem 15. Tag konnte der Patient entlassen werden. Während des Aufenthalts war die Virusinfektion mit SARS-CoV-2 bestätigt worden [2].

Ein weiterer Fallbericht betrifft eine dreiköpfige Familie [11]. Die Mutter fungierte als Auslöser einer innerfamiliären Infektionskette. Primär wurde sie in der Klinik mit einem TCM-Granulat (Jinyebaidu) behandelt, im Verlauf ergänzt durch ein Virostatikum und einen Gyrasehemmer. Bei Zunahme der pulmonalen Symptomatik wurde eine Kombination aus Immuntherapie, Dexamethason und oraler TCM-Flüssigkeit (Shuanghuanglian, SHL) angeordnet und die Patientin letztlich isoliert. Die Tochter wurde 9 Tage später ebenfalls symptomatisch und in Isolation nur mit SHL behandelt. Der Vater war SARS-CoV-2-positiv, jedoch asymptomatisch. Er bekam in häuslicher Quarantäne neben SHL zusätzlich Moxifloxacin und ein Virostatikum. Alle drei Patienten erreichten volle Remission. Die Therapie mit SHL wurde bis zur Entlassung fortgeführt [11]. Auf der Basis dieses Fallberichts ist eine klinische Studie geplant.

Ein weiterer Fallbericht berichtet über 4 Fälle, welche zusätzlich zur antiviralen Medikation (Kaletra ${ }^{\circledR}$ ) ein chinesisches Komplexpräparat (Shufeng-Jiedu-Kapsel) bekamen [14]. Zwei der Patienten wurden im Verlauf negativ getestet und konnten entlassen werden. Bei allen Patienten wurde eine primäre Infektion vermutet, die drei Patienten mit milderen Verläufen profitierten von einer kombinierten Therapie, der vierte Patient in kritischem Zustand zeigte Zeichen der Verbesserung zum Zeitpunkt der Pu-
Hier steht eine Anzeige. Springer 


\section{Johannes Fleckenstein, Eszter Füzeki, Winfried Banzer \\ Die Anwendung der Traditionellen Chinesischen Medizin (TCM)/Akupunktur in der Therapie und Prävention von SARS-CoV-2-Infektionen. White Paper des Wissenschaftszentrums der deutschen Ärztegesellschaft für Akupunktur}

\begin{abstract}
Zusammenfassung
Gegenwärtig kursieren zahlreiche Vorschläge zur potenziellen Wirksamkeit von Traditioneller Chinesischer Medizin (TCM)/Akupunktur in der Therapie von SARS-CoV-2-Infektionen und assoziierten Symptomen. Stand dieser Überlegungen sind insbesondere molekularvirologische, aber auch klinische Untersuchungen aus der SARS-Epidemie 2002/2003. Ein Wirksamkeitsnachweis von chinesischen Arzneimitteln mit antiviralem Potenzial in Bezug auf SARS-CoV-2 steht größtenteils aus. Klinische Daten stützen sich nahezu ausschließlich auf Studien vor Beginn der Coronapandemie. Gegenwärtig finden sich nur international publizierte Fallberichte
\end{abstract}

sowie in China berichtete Beobachtungsstudien. Vielversprechend ist eine große Zahl registrierter prospektiver Studien, die gegenwärtig durchgeführt werden. Die Evidenzlage für die nichtpharmakologischen Therapiesäulen der TCM ist sehr schwach. Auf Basis der Datenlage ist eine Kombination westlicher und TCM-Maßnahmen in der westlichen Welt nur nach Ausschöpfung der konventionellen Maßnahmen gerechtfertigt.

Schlüsselwörter COVID-19 · Schweres akutes respiratorisches Syndrom · Coronavirus . Pandemien · Chinesische Arzneimittel

\section{The use of traditional Chinese medicine (TCM)/acupuncture in the treatment and prevention of SARS-CoV-2 infections. White paper from the Scientific Center of the German Medical Association for Acupuncture}

\section{Abstract}

There are currently numerous suggestions on the potential effectiveness of traditional Chinese medicine (TCM)/acupuncture in the treatment of SARS-CoV-2 infections and their associated symptoms. These reflections are based on molecular virology and clinical investigations resulting from the SARS epidemic in 2002/2003. The antiviral properties of Chinese herbal medicine (CHM) against SARS-CoV-2 have not yet been investigated in depth. Clinical data relies on studies conducted prior to the corona pandemic. Current data is limited to a few internationally published case reports and observational studies published in China. There is a promising number of ongoing registered clinical trials. Evidence regarding TCM treatments other than CHM is poor. Based on the available data, a combination of Western and TCM approaches in the Western Hemisphere seems justified only if all conventional treatments are exhausted.

Keywords

COVID-19 · Severe acute respiratory syndrome - Coronavirus · Pandemics . Drugs, Chinese herbal blikation des Artikels. Die Autoren geben keine Outcome-Parameter an, die einen kausalen Bezug zur chinesischen Arzneimitteltherapie herstellen würden [14].

Randomisiert kontrollierte Studien in etablierten westlichen Fachzeitschriften sind gegenwärtig nicht vorhanden.

Berichte und Untersuchungen zu Nebenwirkungen der chinesischen Arzneimittel oder Kreuzreaktionen mit konventionellen Arzneimitteln sind gegenwärtig nicht vorhanden.

Alle Daten zu TCM/Akupunktur und SARS-CoV-2 wurden bisher in China gewonnen, die Übertragbarkeit auf den westlichen Kontext ist bisher unklar.

\section{Zusammenfassung}

Zum gegenwärtigen Stand steht auf der Basis der Erfahrungen der SARS-Epidemie 2002/2003 im Bereich der TCM insbesondere mit den chinesischen Arzneimitteln ein potenzielles Arsenal antiviraler Wirkstoffe zu Verfügung. Diese sind teilweise molekularvirologisch auf ihre Wirksamkeit hin untersucht. In Bezug auf SARS-CoV-2 stehen diese Untersuchungen jedoch noch größtenteils aus.

Gemessen an der Evidenz klinischer Studien aus den Vorjahren scheint durch eine Kombination mit chinesischer Arzneitherapie mindestens eine Symptomlinderung (Fieber, Husten, Atemnot) erreicht zu werden. Vielversprechend ist die Vielzahl aktuell laufender klinischer Untersuchungen, die diese Wirksamkeit bei COVID-19-erkrankten Patienten untersuchen.

Die weiteren Säulen der TCM, i. e. Akupunktur, Ernährungstherapie, Bewegungstherapie, chinesische Manualtherapie, stehen nicht im Mittelpunkt gegenwärtiger wissenschaftlicher Berichte.

Die gegenwärtige Datenlage rechtfertigt eine additive Therapie mit chinesischen Arzneimitteln, sofern alle konventionellen Maßnahmen ausgeschöpft worden sind. Die A-priori-Kombination westlicher und TCM-Maßnahmen wird durch die Ergebnisse zum gegenwärtigen Zeitpunkt nicht gestützt.

\section{Literatur}

1. National Health Commission of the People's Republic of China (2020) Notice on the issunance of guidelines of diagnosis and treatment for 2019-nCoV infected pneumonia (version 7). http://www.nhc.gov.cn/Xcs/zhengcwj/202003/46c929 4a7dfe4cef80dc7f5912eb1989/files/ce3e6945832a438eaae415350a8ce964.pdf. Zugegriffen: 3. Apr. 2020

2. Deng Z, Yuxing HU, Yang P et al (2020) Diagnosis and treatment of an acute severe pneumonia patient with COVID-19: case report. J Med Virol. https:// doi.org/10.1002/jmv.25802

3. Lai F, Ren Y, Lai C et al (2020) Acupuncture at Zusanli (ST36) for experimental sepsis: a systematic review. Evid Based Complement Alternat Med. https:// doi.org/10.1155/2020/3620741 
Tab. 1 Empfohlene chinesische Arzneimittel. (Modifiziert nach [1, 15, 16].) Eine Anpassung an lokale und individuelle Gegebenheiten darf durch geschulte Anwender erfolgen

\begin{tabular}{|c|c|c|}
\hline Stadium der Erkrankung & Symptom & Empfehlung (patentierte Rezepturen) \\
\hline \multirow{2}{*}{$\begin{array}{l}\text { Medizinische Beobachtung } \\
\text { (z. B. häusliche Quarantäne, unbestätigt) }\end{array}$} & Fatigue mit gastrointestinalen Beschwerden & Huo Xiang Zheng Qi Wan (Fertigarznei) \\
\hline & Fatigue mit Fieber & Fertigarzneien auf Yin-Qiao-San-Basis \\
\hline \multirow{5}{*}{$\begin{array}{l}\text { Klinische Behandlung } \\
\text { (positiv getestete Patienten) }\end{array}$} & Allgemein & Qing Fei Pai Du Tang (Dekokt) \\
\hline & Mild & $\begin{array}{l}\text { Han Shi Yu Fei Zheng (Dekokt) } \\
\text { Shi Re Rao Fei Zheng (Dekokt) }\end{array}$ \\
\hline & Moderat & $\begin{array}{l}\text { Han Shi Zu Fei Zheng (Dekokt) } \\
\text { Shi Du Yu Fei Zheng (Dekokt) }\end{array}$ \\
\hline & Schwer & $\begin{array}{l}\text { Yi Du Bi Fei Zheng (Dekokt) } \\
\text { Qi Yin Liang Fan Zheng (Dekokt) }\end{array}$ \\
\hline & Kritisch (Beatmung) & $\begin{array}{l}\text { Ren Shen, Hei Shun Pian, Shan Zu Yu } \\
\text { Su-He-Xiang-Tablette, An-Gong-Niu-Huang-Tablette }\end{array}$ \\
\hline
\end{tabular}

4. Leung PC (2007) The efficacy of Chinese medicine for SARS: a review of Chinese publications after the crisis. Am J Chin Med 35:575-581

5. LiC, Wang P, Zhang L et al (2018) Efficacy and safety of Xuebijing injection (a Chinese patent) for sepsis: a meta-analysis of randomized controlled trials. J Ethnopharmacol 224:512-521

6. Li T, Peng T (2013) Traditional Chinese herbal medicine as a source of molecules with antiviral activity. Antivir Res 97:1-9

7. Ling CQ (2020) Traditional Chinese medicine is a resource for drug discovery against 2019 novel coronavirus (SARS-CoV-2). J Integr Med 18:87-88

8. Liu J, Manheimer E, Shi Y et al (2004) Chinese herbal medicine for severe acute respiratory syndrome: a systematic review and meta-analysis. J Altern Complement Med 10:1041-1051

9. Liu X, Zhang M, He L et al (2012) Chinese herbs combined with Western medicine for severe acute respiratory syndrome (SARS). Cochrane Database Syst Rev. https:// doi.org/10.1002/14651858.CD004882.pub3

10. Luo H, Tang QL, Shang YX et al (2020) Can Chinese medicine be used for prevention of corona virus disease 2019 (COVID-19)? A review of historical classics, research evidence and current prevention programs. Chin J Integr Med 26:243-250

11. Ni L, Zhou L, Zhou M et al (2020) Combination of western medicine and Chinese traditional patent medicine in treating a family case of COVID-19 in Wuhan. Front Med. https://doi.org/10.1007/s11684-020-0757-X

12. Ren JL, Zhang AH, Wang XJ (2020) Traditional Chinese medicine for COVID-19 treatment. Pharmacol Res 155:104743

13. Wan S, Xiang Y, Fang Wet al (2020) Clinical features and treatment of COVID-19 patients in northeast Chongqing. J Med Virol. https://doi.org/10.1002/jmv.25783

14. Wang Z, Chen X, Lu Y et al (2020) Clinical characteristics and therapeutic procedure for four cases with 2019 novel coronavirus pneumonia receiving combined Chinese and Western medicine treatment. BST 14:64-68

15. Wiebrecht A (2020) CTCA Letter Sonderausgabe März 2020: Chinesische TCMBehandlungsempfehlungen zu Covid-19. https://www.ctca.center/de/newsletter/ ctca-letter-sonderausgabe-maerz-2020.html. Zugegriffen: 3. Apr. 2020

16. Yang Y, Islam MS, Wang J et al (2020) Traditional Chinese medicine in the treatment of patients infected with 2019-new Coronavirus (SARS-COV-2): a review and perspective. Int J Biol Sci 16:1708-1717

\section{Korrespondenzadresse}

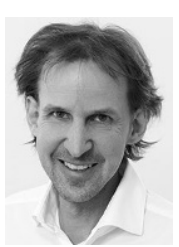

PD. Dr. Johannes Fleckenstein

Institut für Komplementäre und Integrative Medizin

IKIM, Dozentur für TCM/Akupunktur,

Universität Bern

Freiburgstr. 46, Haus 4, 3010 Bern, Schweiz

johannes.fleckenstein@ikim.unibe.ch

\section{Einhaltung ethischer Richtlinien}

Interessenkonflikt. J. Fleckenstein, E. Füzeki und W. Banzer geben an, dass kein Interessenkonflikt besteht. Die Mitglieder des Wissenschaftszentrums erhalten eine Aufwandsentschädigung seitens der Deutschen Ärztegesellschaft für Akupunktur e. V.

Für diesen Beitrag wurden von den Autoren keine Studien an Menschen oder Tieren durchgeführt. Für die aufgeführten Studien gelten die jeweils dort angegebenen ethischen Richtlinien. 